\title{
Taiwanese Fathers' Experiences of Having Their Child Diagnosed With a Developmental Disability
}

\author{
Yu-Ping Huang ${ }^{1} \&$ Winsome St John ${ }^{2} \&$ Sen-Wei Tsai ${ }^{3} \&$ Hsiu-Jung Chen ${ }^{4}$
}

1. RN, PhD, Assistant Professor, Department of Nursing, Hung Kuang University \& 2RN, PhD,

2. Associate Professor, School of Nursing and Midwifery, Research Centre for Clinical and Community Innovation, Griffith University, Brisbane, Australia

3. MD, Attending Physician, Rehabilitation Department, Taichung Veterans General Hospital

4. RN, PhD, Associate Professor, Department of Nursing, Hung Kuang University.

Citation:

Huang, Y., St John, W., Tsai' S., \& Chen, H. (2011). Taiwanese fathers' experiences of having their child diagnosed with a developmental disability. Journal of Nursing Research, 19(4), 239-249.

Accepted for publication: August 22, 2011.

Address correspondence to: Yu-Ping Huang, No. 34, Chung-Chie

Rd., Sha Lu District, Taichung City 43302, Taiwan, ROC.

Tel: +886 (4) 2631-8652 ext. 7050; Fax: +886 (4) 2633-1198;

E-mail: yup@sunrise.hk.edu.tw

DOI: 10.1097/JNR.0b013e3182389e34 


\begin{abstract}
Background:

Receiving a diagnosis of a developmental disability in a child can be a crisis event for parents. Gender differences in parental roles are worth considering when exploring the impact of having a child with a disability. However, most studies on this topic have focused on the mother's experience, and little is known about what the father goes through as the parent of a child diagnosed with a disability. Even less is known regarding this experience in the context of the Chinese culture.

Purpose: The goal of this study was to explore fathers' experiences of having a child diagnosed with a developmental disability in a Chinese cultural context.

Methods: This study used a hermeneutic phenomenological approach informed by the philosophical world views of Heidegger. The 16 fathers who participated in the study were purposively sampled from a teaching hospital in central Taiwan. Data were collected using in-depth and semi structured interviews and were analyzed using hermeneutic analysis.
\end{abstract}

Results: Data analysis revealed four shared meanings: losing hope, feelings of failure, being frustrated with family conflicts, and searching for positive coping strategies.

Conclusions/Implications for Practice: Fathers feel shock and despair as well as personally devalued when learning that their child has been diagnosed with a developmental disability. Chinese cultural beliefs and values can elicit different experiences for fathers while helping them make sense of their experiences and accept their child in meaningful ways. Nurses can actively engage fathers as well as mothers to understand their feelings and thoughts about their child's disability to provide appropriate emotional and informational support. Providing support or referral is necessary particularly when fathers encounter issues with the child's grandparents. Nurses can assist fathers to find a way to make sense of having a child with a disability within their cultural frame of reference by adapting cultural beliefs and values to their situation and to make meaning of their child's life.

KEY WORDS: developmental disability, child, diagnosis, fathers, nursing, phenomenology 


\section{Introduction}

Becoming a parent is an important milestone for men and women. Parents begin building a relationship with their child before a baby is born (Clark \& Miles, 1999). An expectant woman and her partner start to build an image of their "wished for" or "ideal and perfect" baby as they see ultrasound images and physical changes. Parents usually anticipate that their baby will be healthy and will develop in ways that they expect. When a baby is born with an impairment or disability, parents experience shock, disbelief, fear, uncertainty, loss of control, anxiety, and grief due to losing their "perfect infant" (Graungaard \& Skov, 2006; Huang, Kellett, \& St John, 2010; McGrath, Paton, \& Huff, 2004).

A child with a disability can negatively impact family functions, leading to poor communication among family members, emotional bewilderment for the siblings, and the child as a source of sorrow (Chang \& McConkey, 2008). In addition, a child's diagnosis of disability is a crisis event for parents, and gender differences in parental roles should be considered when exploring the impact on parents of having a child with a disability (Premberg, Hellstrom, \& Berg, 2008). However, most studies on parents' experiences of having a child with a disability have focused on mothers (Huang et al., 2010; Mri \& Tovey, 2003), although some researchers have included fathers together with mothers. For example, 14 parents (5 fathers and 9 mothers) of children with developmental disabilities in the United Kingdom were found to experience an inner emotional journey, including feeling shock and devastation, feeling upset and traumatised when the child was undergoing continuing tests, wanting to have a second diagnosis, and searching for treatment so that they could anticipate the future (Lewis, Skirton, \& Jones, 2010).

In another study, 13 mothers and 13 fathers in Canada were found to help each other adapt to the changes in their lives, including their relationship with one another, parental roles, and relationships outside the family (Pelchat, Levert, \& Bourgeois-Gue'rin, 2009). Some fathers withdrew and kept their distance from dealing with their child's diagnosis (Pelchat et al., 2009). Similarly, 8 mothers and 9 fathers in Sweden were found to have both positive and negative emotions toward their child with a disability (Bostro"m, Broberg, \& Hwang, 2010). These parents' attitudes toward their child's diagnosis varied from preoccupation with the past to acceptance, where they moved freely between past and present events and toward hope for the future (Bostro"m et al., 2010). On the other hand, 16 parents of children with disabilities in Denmark were found to search for diagnostic certainty during the diagnostic processes, which induced feelings of uncertainty about the future (Graungaard \& Skov, 2006).

Although these studies identified issues for fathers, only small numbers of fathers were included, and their voices were mixed with those of mothers. Fathers' voices should be separated from those of mothers to let their inner words be heard (Premberg et al., 2008). Focusing only on mothers and ignoring fathers may hinder fathers' engagement with their child's care and encourage traditional or detached fathering roles. In addition, these studies focused on parents in Western countries. Because perceptions of disability are greatly influenced by culture (Liu, 2001), and little is known about fathers of children with disabilities in Chinese cultures, it is important to study this phenomenon in a Chinese context. Furthermore, Chinese fathers play a crucial role in families because the Chinese society is paternalistic, and fathers usually have the highest positions of power and authority within the family (Lee, 1999). Therefore, fathers have considerable influence on the family, especially when a child is diagnosed with a disability. Providing for the family financially has long been regarded as the most important role for fathers. However, fathers are expected to be more involved in their children's care to contribute to a positive child-father relationship, improve the quality of childcare, and improve the marital relationship (Keller \& Honig, 2004). Better understanding of fathers' experiences would enable healthcare professionals to provide more appropriate support and care. Furthermore, involving fathers in their child's care could enhance appropriate family centred care in terms of involving mothers and fathers rather than mothers only. Thus, the goal of this study was to explore fathers' experiences of having their child diagnosed with a developmental disability in the context of Chinese culture.

\section{Methods}

\section{Design}

This study used a hermeneutic phenomenological design due to its ability to describe human experiences and discover their meaning and provide the holistic context of fathers' experiences 
(Wojnar \& Swanson, 2007). Hermeneutic phenomenology is informed by the philosophical world views of Heidegger (1927/1962) and Gadamer (1960/1975). Heidegger provided guidance to explore the meaning of fathers' daily experience of having their child diagnosed with a disability, whereas Gadamer helped the researchers understand and interpret the meaning of stories shared by participant fathers.

\section{Participants}

This study was a component of a larger study exploring fathers' experiences of having a child with developmental disability. Fathers were purposefully recruited from a teaching hospital in central Taiwan. Inclusion criteria were as follows: (a) having a child (0Y18 years old) with a developmental disability, (b) living in the same household with the child with a developmental disability, (c) speaking Mandarin, Taiwanese, or Hakka, and (d) being willing to participate in this study. For Criterion a, the wide age range was chosen because it mirrored the range of paediatric clients as defined in Taiwan (Chen, 2008). Further, this range of ages was also chosen based on Heidegger's assumption that time should be considered in terms of past, present, and future to understand the meanings of the human experience. The event of their child's diagnosis was a past experience for fathers. Such a meaningful event would connect the past with the present and future (Kellett, 1997). This study did not focus on one specific diagnosis, disability, or age because the focus of this study was on the meanings that fathers ascribed to having a child with a disability rather than on the diagnosis itself or comparing fathers' experiences across different disabilities or children's ages.

Fathers were invited to join this study either via direct contact in the visiting room of the hospital or rehabilitation setting or by asking mothers to take the research information package home to fathers. Three fathers were contacted directly in the visiting room, five fathers were asked to participate during visits to the rehabilitation setting, and eight fathers were contacted by telephone after mothers had taken an information package home. No children with disabilities were hospitalised while participating fathers were interviewed. Eight fathers were interviewed in their homes and eight were interviewed in a hospital conference room.

\section{Data Collection}

Before data were collected, the study received ethics approval from the participating hospital, and all participants signed and returned informed consent before being interviewed. Each of the 16 fathers participated in two in-depth interviews conducted by the first author between March and December 2009. Initial interview questions were broad and open to gain a picture of fathers' experiences, for example, "Could you please tell me about your experience when you learned that your child had a disability?" Probe questions were used to explore issues raised by fathers, for example, "Can you give me more details about that?" Journal notes were taken immediately after each interview to serve as an audit trail (Koch, 1998). Data were analyzed concurrently with data collection. Follow-up interviews served to clarify information as well as to confirm fathers' agreement about the interpretation of their experiences. Interviews lasted 50 to 100 minutes, and all were audio-recorded. Data collection ceased after 32 interviews with 16 participants as data saturation was reached and no new information was emerging.

\section{Data Analysis}

All audio-recorded interviews were transcribed verbatim. Transcripts and journal notes were analysed to capture the meaning of the fathers' experiences and to achieve thick description by using a hermeneutic circle (Palmer, 1969). Thick description means that fathers' thoughts and feelings can be depicted and described deeply and richly within their particular context. Thus, essential meaning and elements of their experiences and findings can be understood comprehensively (Ponterotto, 2006). Gadamer (1960/1975) argued that people can realize a particular historical moment and context because of their horizon. Thus, researchers' interpretations are influenced by their personal prejudgments and previous beliefs, that is, their past horizon. However, to deeply understand a text and more accurately interpret it, researchers should go beyond their past horizon to a new horizon to fuse these two horizons. Therefore, the process of understanding participant stories involves a "fusion of horizons" (Clark, 2008; Gadamer, 1960/1975).

Data analysis involved first listening to audio-taped interviews and reading and re-reading interview transcripts. Next, a summary was written for each interview transcript, including main impressions and initial interpretations of fathers' experience of having their child diagnosed with disability, which elicited possible meanings. The next step was to turn to the parts of stories, which meant that 
transcripts were coded line-by-line and labelled to find significant paragraphs or sentences related to the study purpose in terms of parts of stories. Shared meanings were thus developed, which helped the authors gain insight into the fathers' experiences. Hermeneutic interpretation was the final step of data analysis. The authors moved back and forth between whole stories and parts (shared meanings) to gain a deeper understanding of fathers' experiences (Palmer, 1969).

\section{Trustworthiness}

The researchers enhanced the rigour of findings using the following four criteria: credibility, dependability, transferability, and confirmability (Lincoln \& Guba, 1985). Credibility was achieved by the first author's extensive experience (14 years) in the field of paediatric disability care. Credibility was further enhanced by member checks with the 16 participants during the second interview and by inviting experts in the phenomenological approach to validate emerging themes. Peer debriefing was applied to the whole research process, including data analysis and findings, to ensure better understanding and interpretation of participant stories. Dependability was confirmed by recording details of the research process in terms of recruiting participants, data collection, and data analysis to allow others to conduct similar research. Transferability and confirmability were confirmed by recording detailed journal notes, transcribing data verbatim, and keeping an audit trail of details related to the research process (Lincoln \& Guba, 1985).

Journal notes functioned as an audit trail to help the authors make decisions related to the research process. Such notes included contextual documentation (recording participants' verbal and nonverbal communication and the scene of the interview), methodology documentation (recording how to recruit potential participants and research techniques applied in this study), analytic documentation (recording how data were analysed), and personal response documentation (recording the interviewer's thoughts, feelings, and reflections; Rodgers \& Cowles, 1993).

\section{Results}

\section{Characteristics of Participating Fathers}

The fathers in this study ranged in age from 32 to 48 years (mean age $=37.3$ years), and their education level ranged from senior high school to college. The age of the children with disabilities ranged from 18 months to 10 years. See Table 1 for characteristics of the disabled children.

Analysis revealed that the fathers' experiences of having their child diagnosed with a developmental disability were associated with four shared meanings: losing hope, feelings of failure, being frustrated with family conflicts, and searching for coping strategies.

\section{Losing Hope}

A healthy child gives fathers hope because such a child can help ensure future success and happiness - both for the child and the family as a whole. Fathers lost hope because they lost their anticipated "ideal" child and worried about the future of the child with a disability and their family.

\section{Loss of the ideal child}

Fathers felt overwhelming shock and extreme sadness because their anticipated ideal child or present reality was destroyed by their child's diagnosis of disability. This loss of their ideal child was experienced in three situations: the child was diagnosed at birth, the child became disabled by an unexpected medical event, and the child's disability was gradually revealed through related behaviours and symptoms. First, when the child was diagnosed with a disability at birth, fathers' hopes and dreams for their new babies and families were dashed. Fathers could see no future and felt overwhelming shock and panic. Father 8 said,

My child experienced a difficult delivery and meconium aspiration. In addition, [when the child was born] he had no heartbeat or respiration at all. He therefore was sent directly to the neonatal intensive care unit. It happened so suddenly. At that moment, I panicked and felt like it was the end of the world. When my wife was pregnant, we were so happy and looked forward to having a healthy baby, but it did not happen this way.

Second, some fathers lost their ideal child when their healthy child became disabled due to unexpected medical events, such as an enterovirus infection or stroke. Fathers originally had a clear and concrete image of their healthy child. When their child experienced severe and dramatic changes, fathers reacted with shock, denial, and fear for the future. Father 1 said, 
My daughter could walk and talk at about 1 year old. She was very smart and learned things very quickly. I could tell she would be a future leader. When she was about 1 year [old], she went suddenly into shock after having a high fever and wasn't able to breathe properly. We rushed her to a hospital, but she hasn't able to move from that time to now. Il felt deeply hurt and hugely overwhelmed after seeing that. I couldn't fall asleep as I used to, except after taking sleeping pills or drinking. I have tried to stop doing that, but I just can't.

Third, some fathers gradually became aware of their child's disability as the child's development or behaviours gradually deviated from the norm. Fathers gave time to their child in the hope that he or she would be "normal." Fathers' hopes were completely destroyed when they were received a formal diagnosis, which caused suffering and negative feelings. Father 7 said,

When my son was about 1 year old, we found that he often wasn't able to interact with others. Children his age usually like to play with people. He avoided looking at us and had no eye contact. He especially likes to play with toys with wheels or balls. We were worried about it but still kept waiting and hoping that he would be OK after he grew up. We took him to see a doctor when he was 2, and the doctor told us that my son's diagnosis was autism. No one could accept it ... me either.

Participants experienced several stages of emotional reactions toward the loss of their ideal child. After first learning the diagnosis, fathers felt shock and denied their child's disability. After diagnosis, fathers felt anxiety and panic about the situation. Then they questioned the diagnosis and spent time testing the diagnosis in terms of giving time to their child with a disability, hoping or wishing that their child with a disability would be revealed as a normal child. When the diagnosis could not be changed, fathers experienced despair and depression. However, fathers' stages of emotional reactions to their child's diagnosis or disability could be interwoven, without any clear cut transition or distinction between stages.

\begin{tabular}{|c|c|r|c|}
\hline No. & Age & Diagnosis & Age at diagnosis \\
\hline 1 & 9.0 & Cerebral palsy & 18 months \\
\hline 2 & 10.0 & Joubert syndrome & 8 months \\
\hline 3 & 1.6 & Neurological sequelae of infection with enterovirus 71 & 9 months \\
\hline 4 & 7.0 & Cerebral palsy & 4 months \\
\hline 5 & 1.5 & $\begin{array}{r}\text { Tetralogy of Fallot (serious loss of physical functions } \\
\text { after heart surgery at 8 months old }\end{array}$ & right after birth \\
\hline 6 & 2.4 & Down's syndrome & right after birth \\
\hline 7 & 3.0 & Autism & 2 years \\
\hline 8 & 3.0 & Cerebral palsy & 3 months \\
\hline 9 & 6.0 & Spino-cerebellar ataxia & right after birth \\
\hline 10 & 3.0 & Cerebral palsy & 10 days \\
\hline 11 & 3.0 & Hydrocephaly & 10 months \\
\hline 12 & 2.0 & Neurological sequelae of infection with enterovirus 71 & 9 months \\
\hline 13 & 2.0 & Cerebral palsy & 3 years \\
\hline 14 & 7.0 & Stroke & Right after birth \\
\hline 15 & 9.0 & Chromosomal abnormality & 2 months \\
\hline 16 & & Cerebral palsy & \\
\hline
\end{tabular}

TABLE 1: 1.7 Characteristics of the children with disabilities

\section{Worrying about the future}

From the moment of diagnosis, fathers felt unable to look forward to a positive future because the disability was linked to negative images of deficient abilities. Fathers felt uncertain about what sort of future could be anticipated for their child with a disability and their family. Father 10 said,

I felt very stressed, because he was my first child and he has problems. He is the eldest grandson in our family, so his responsibility for our family should be very heavy. Like me. I am the first son in my family. So I need to make decisions on family matters or be responsible for my parents and so on. II am so worried about whether he can shoulder such responsibility in the future. Thus, I hope he [the disabled child] can catch up with his development and grow up well. 
Worry about the future was sometimes made more difficult when family members, especially grandparents who have a powerful social position in the family, were concerned. Negative appraisals from grandparents affected fathers profoundly because of their close relationship and kinship. Father 7 said,

My parents think my son with problems is unable to achieve anything or do anything well in the future. I felt very sad when I heard them say that.

\section{Feelings of Failure}

Fathers felt a sense of failure because they not only blamed themselves for their child's disability but also felt devalued personally and socially. This theme had two subthemes: self-blame for inappropriate fathering and feelings of lower self-evaluation.

\section{Self-blame for inappropriate fathering}

Fathers had doubts about their ability to protect their child from harm and to provide for his or her well-being. Fathers often blamed themselves for their child's disability because they thought that they had not fulfilled their obligation to watch over their child. Father 12 said,

I thought that [the disability] was my fault because my daughter was exposed to germs due to my carelessness and negligence. She didn't do anything wrong. If she cannot fully recover and walk well in the future, then I will have ruined her life. People say, "Men don't weep easily." But I can't help crying when I am alone.

Some fathers blamed themselves for not making the best daily life choices for their child. For example, Father 3 said,

I chose a baby sitter whose place was close to my house so I could drive my son and pick up him on the way to work. However, my wife didn't like that place because the baby sitter looked after four kids, including my son. My son was contaminated by enterovirus from that crowded place. I blame myself fully for not listening to my wife's suggestion to choose a better place for my son.

Other fathers blamed themselves for the choices they made about medical care. Father 5 said, Every hospital brags about their advanced and modern technology. But I now regret having chosen a regional hospital instead of a medical centre where doctors might have diagnosed my son's problem even earlier.

\section{Feelings of lower self-evaluation}

Feelings of lower self-evaluation meant that fathers made negative judgements about their own personal and social qualities because they viewed the child with a disability as part of themselves, especially when their child was born with a physical deformity. Father 15 said,

When my son was born, the doctor told me that my son had a strange face, so there might be some problems with his chromosomes. That meant my son got those problems because of my wife and me. I felt very guilty and sad after the blood test that found my son's first pair of chromosomes had problems.

Other fathers believed that their child's disability was retribution for something they might have done wrong in their previous or present life. Father 12 said,

My daughter underwent an operation to repair her intestine after an enterovirus infection. When I heard the doctor explain the surgical procedure to me, I was really scared. Was this retribution for my selling pork?

Participants felt that their child's disability hindered their social life due to feelings of shame and misfortune. Father 1 said,

It's not an honourable thing to talk about my daughter's story to others. In addition, if I did so, the atmosphere would become frozen suddenly. Only a few friends know my daughter's story. I'm also afraid that someone will ask me about my daughter, so I don't like to attend social activities like I used to do, especially weddings or celebrations for a new baby. My mood becomes very bad after attending those kinds of ceremonies.

\section{Being Frustrated With Family Conflicts}


The participants had to manage family arguments and conflicts related to the child's disability, which they found frustrating. These conflicts made many delay seeking help to manage their child's medical needs.

\section{Struggling with their child's medical needs}

Fathers struggled when opinions and interpretations of their child's development or behaviours differed with those of their parents. Fathers were particularly concerned when the grandparents denied their grandchild's developmental disability, leading to delayed management of the child's medical needs. Father 10 said,

My son wasn't able to sit when he was 7 months old. I wanted to bring him to the hospital for an examination, but my mother thought that my son's development was just a little bit late for his age and he would be fine when he grew up. She couldn't accept that my son has a deficit. I just kept waiting to avoid arguing with my mother, so we didn't go to hospital for an examination. I brought my son to hospital for an examination after an argument with my mother. By that time, my son was 9 months old and his condition didn't improve with time.

However, some fathers insisted on maintaining their own ideas when arguing with their parents, thus disrupting their relationship. Father 7 said,

When he was 2 years old, he couldn't speak a single word or make eye contact with others. I wanted to bring my son to the doctor for a consultation. However, my father stopped me from doing so. He couldn't accept that my child is autistic. But my mind was made up since my son's condition was really strange. During this time I was quarrelling with my father. I needed to force myself to deal with this situation.

\section{Managing family arguments}

Fathers also had to deal with family arguments between their own blood relatives and relatives on their wife's side of the family. The arguments centred on the family's reputation, which was perceived as connected to the honour of their lineage. Fathers felt powerless and helpless because of their inability to control the arguments, particularly because family cohesion and harmony are a top priority for traditional Chinese families. Father 7 said,

My father said, "How could we have a child with autism in our family?" My father concluded that all his children and grandchildren are normal, except my son. So my father thought the problem came from my wife's family. But my wife argued that my son's problems didn't come from her family. Everyone just argued and argued over whose family should be responsible for my son's problem.

Another family issue was that some grandparents requested fathers to have another "healthy and normal" child to regain the family's reputation. This implied rejection or denial of the child with a disability. Fathers felt that this attitude treated the child with a disability as an incomplete human being. Father 14, who was the only male in his family, said,

My mom asked me to have another child after my son got sick. I felt sad. If I wanted to have another child that meant I gave up on him. If I had another child, the new baby might get all my attention, so my son wouldn't have the best care from me. That bothers me quite a bit. We have this kind of argument every now and then.

\section{Searching for Positive Coping Strategies}

Fathers gradually accepted their child with a disability. The time for fathers to accept their child with a disability varied according to fathers' perceptions and adjustment. However, fathers were helped in this adjustment process by two important strategies: seeking help from health professionals and developing a positive perspective and meaning.

\section{Seeking help from health professionals}

Health professionals were an important source of help for participants to cope with their child's diagnosis of disability. The health professionals' knowledge and authority gave fathers support to deal with their difficulties. Father 16 said,

I let the doctor tell my wife about our child's problems, even though I was also standing right beside her. The doctor is a professional, and his explanation was much clearer than mine. Also the doctor was a witness when it [the process of childbirth and resuscitation] happened. 
In addition, fathers solved familial conflicts by using health professionals' authority and reassurance. Father 10 said,

The doctor told me that my child could apply for a handicapped certificate to receive government and social assistance. But my mom didn't want my son to have it. I asked the doctor to explain my child's situation to her. The doctor did it for me. The doctor explained that the certificate would be cancelled when my son's development caught up to his age, and it wouldn't follow my son forever. My mom finally accepted the doctor's suggestions.

\section{Developing a positive perspective and meaning}

Having their child diagnosed with a disability had a negative impact on fathers, but it also provided them with opportunities to rethink how this situation/experience was meaningful to them and to rearrange their life priorities. Father 14 said,

I saw my son falling down in front of me [the child lost consciousness due to a stroke while playing with the father outside their house] and suddenly was unable to move at all. I thought that I could give up all other things to gain my son's health back. The only thing I thought of was to take good care of him and spend more time with him. So I have started to decrease my work hours and I have decreased my amount of business in order to have more time to take care of him.

Fathers also found positive meaning in having a child with a disability based on the traditional Chinese saying "Yuan fen brings two people together." They viewed their responsibility as a sweet duty rather than a burden. Father 2 said,

After learning that my child had Joubert syndrome, I thought, "Why do I have this kind of child?" II have changed my way of thinking. Now I think he became my son because of predestination. I should take him into my heart and pay more attention to him, not just focus on my negative feelings.

Using old traditional Chinese sayings helped fathers to believe that the disability was a blessing in disguise and enabled them to regain control and hope over the situation. Father 6 said, Old people say that this kind of child will bring fortune to the family. Honestly, my company's business has really done better since my son was born.

Father 8 , who has a child with severe cerebral palsy due to anoxia during birth, said,

I believe that my son will have a fortunate life because he is alive after such a huge disaster.

\section{Discussion}

Fathers lost hope due to their child's disability, with initial feelings of sadness and shock, and felt that the diagnosis was the "end of the world." This finding is supported by previous studies on parents of children with disabilities (Hill, Sahhar, Aitken, Savarirayan, \& Metcalfe, 2003; Kerr \& Mclntosh, 1998; Makela, Birch, Friedman, \& Marra, 2009). Parents' emotions fluctuated frequently and erratically after learning of their child's diagnosis (Hill et al., 2003). Having a child with a disability led parents to experience sudden and drastic changes in their lives, as their baby and parenting roles were not as they had expected. They grieved over the loss of their imagined perfect child (Schuengel et al., 2009). In Chinese culture, parents emphasize the next generation's "good" qualities that are required for a successful future, including growing up healthy with a well-rounded morality, intelligence, ability, physical appearance, and beauty (Hwang, 1987). Children can promote their parent's social status and prestige, give them "face" in their social networks, and bring them a better quality of life. These fathers had to find new ways of thinking about their child that made sense of their child's life.

Many fathers in this study thought that they were responsible for their child's disability and felt shame, guilt, and low self-evaluation, which are rooted in the traditional Chinese view of a person with a disability as useless and a burden (Liu, 2001) as well as the concept of losing face (Lai, 2006). A disability in traditional Chinese culture is sometimes viewed as punishment from the gods for a person's transgression in the previous or present life (Lam, Tsang, Chan, \& Corrigan, 2006). Similarly, ethnic Chinese living in Australia still believed in retribution, that is, that past or current misdeeds could cause disease or ill health for the present and future generations (Yeo et al., 2005). Thus, fathers in this study blamed themselves for inappropriate fathering and for not protecting their child from harm, consistent with previous findings (Harden, 2005; Pelchat et al., 2009). Some fathers in this study felt too ashamed to talk about their child with a disability with others or to attend public 
functions, which negatively affected their social lives. This finding is consistent with previous reports that parents felt strain in building up their social networks because of their child with a disability and lack of acceptance by others (Lewis et al., 2010) and worried very much about other people's views of their child with a disability (Bostro"m et al., 2010).

Fathers in this study faced conflicts with their own parents, which upset familial harmony and order by challenging grandparents' authority in the family. Challenging traditional beliefs within the family can potentially lead to a loss of familial support. In Chinese culture, people are expected to behave in accordance with the expectations of significant others and to love and respect their parents. Fathers in this study felt upset and powerless when their own parents denied the value of their child with a disability. This finding echoes reports that Chinese fathers of children with disabilities in Hong Kong would pay any price in order to have a normal son to regain their reputation (Holroyd, 2003) and that Australian Chinese parents of children with disabilities felt sorrow when their families projected feelings of isolation, rejection, hurt, and failure (Kearney \& Griffin, 2001). In contrast, McNeill (2004) found that having a child with a disability can bring family members closer, especially in the diagnosis stage. These findings highlight the importance of social support, family involvement, and acceptance to help parents to deal with difficulties related to a child's disability (Makela et al., 2009), particularly at the diagnostic stage.

Fathers in this study tried to guide the attitude of others to their child's diagnosis of disability by seeking help from health professionals, who gave fathers information and emotional support and assisted them with family conflicts. However, another study found that Taiwanese parents of a child with a learning disability believed that support from family and friends was more important and useful than that from professionals (Chang \& Hsu, 2007). A "good" physician in Chinese society is expected to treat not only the disease but also the patient's heart (Lee, 1999). Chinese people tend to regard physicians as problem solvers because of their professional medical knowledge and ability to provide a cure, which gives them a superior social status and extreme authority and power when giving medical diagnoses, advice, and treatment (Lee, 1999; Yip, 2004). Fathers in this study developed a positive perspective and value from their experience of having a child with a disability. This finding was supported by previous reports that parents of children with disabilities from the United Kingdom (Harden, 2005) and Canada (McNeill, 2004) found ways to think positively and make life as normal as possible. Similarly, parents of children with disabilities in the United Kingdom tried to accept their child's condition and normalize their lives so that their child's disability did not perpetually influence their lives (Lewis et al., 2010). Likewise, Canadian parents (Pelchat et al., 2009) regarded having a child with a disability as an opportunity for personal growth, allowing them to overcome such challenges and adapt successfully. In Chinese culture, fathers should heed social expectations of being a "good" father by resuming their parental responsibility to overcome difficulties and not abandon the child with a disability (Park \& Chesla, 2007). Some fathers also believed that they could become a better person through their children's disabilities (Park \& Chesla, 2007), basing these changes in traditional Chinese wisdom.

When fathers received their child's diagnosis, they were aware of how their situation of anticipated fatherhood had changed when they became the father of a child with a disability. Such a change had a profound impact on fathers and their significant others, which can include alterations in fathers' identities, roles, relationships, abilities, and behaviours (Schumacher \& Meleis, 1994). After becoming aware of these changes, fathers were able to give meaning to and develop new expectations. That adjustment helped fathers ease their stress and negative emotions attributable to the transition process. Support and effective communication from people around fathers can help them better manage the transition with better resultant outcomes. Fathers in this study gave positive meanings to their child's diagnosis, developing a positive perspective and seeking support from health professionals. However, negative appraisal of the child's disability from grandparents, family conflicts, or societal attitudes (stigmatization or marginalization) could hinder fathers' finding positive meaning in their child's life (Meleis et al., 2000; Schumacher \& Meleis, 1994).

\section{Research Limitations and Suggestions}

Taiwanese fathers of a child with a developmental disability in Chinese society are greatly influenced by their parents. Thus, we suggest further studies to explore grandparents' experiences of having a grandchild with a disability. Fathers in this study were Taiwanese, so we suggest further research to focus on immigrant fathers of children with disabilities. The period since the child's diagnosis varied among participants, which influence perceptions of experiences. Future studies should be defined to 
interview fathers at specific times in their child's life. This study did not focus on a specific diagnosis or disability, so future studies may explore fathers' experiences after diagnosis with a specific disability. This study did not analyse various factors influencing fathers' experiences of having their child diagnosed with a disability, such as the child's siblings. Thus, future studies could explore how different family conditions and situations impact father experiences.

\section{Conclusions}

Results found that a diagnosis of disability in their children caused fathers to not only lose hope and feel uncertain about the future but also to blame themselves for inappropriate fathering practices and feel shame and guilt for their child's diagnosis. A disability diagnosis was found to also be a source of family arguments and conflict. Finally, fathers were able to give meaning to their child's diagnosis and use health professionals as part of a support strategy.

Nurses should recognise that fathers feel shock, sorrow, and despair when their child is diagnosed with a disability. In this situation, nurses can actively engage fathers and mothers to understand their feelings and thoughts about their child's disability to provide appropriate emotional and informational support. Providing support or referrals is necessary, particularly when fathers encounter issues with the grandparents of the child with a disability. Nurses may be able to assist fathers to find a way to make sense of having a child with a disability within their cultural frame of reference and by adapting cultural beliefs and values to their situation and to make meaning of their child's life. Study findings show that nurses can assist and encourage fathers to appreciate their child's positive capabilities and use positive thinking to help themselves face issues related to the child's disability. Importantly, although Chinese cultural beliefs and values can make fathers' experiences difficult, they can also provide a way of making sense of their experiences and accepting their child in meaningful ways.

\section{References}

Bostro" m, P. K., Broberg, M., \& Hwang, P. (2010). Parents' descriptions and experiences of young children recently diagnosed with intellectual disability. Child: Care, Health, and Development, 36(1), 93-100.

Chang, M. Y., \& Hsu, L. L. (2007). The perceptions of Taiwanese families who have children with learning disability. Journal of Clinical Nursing, 16(12), 2349 -2359.

Chang, M. Y., \& McConkey, R. (2008). The perceptions and experiences of Taiwanese parents who have children with intellectual disability. International Journal of Disability, Development and Education, 55(1), 27-41.

Chen, Y. C. (2008). Introduction. In Y. C. Chen (Ed.), Practical pediatric nursing (1st ed., pp. 1-32). Taichung, Taiwan: Wagner. (Original work published in Chinese)

Clark, J. (2008). Philosophy, understanding and the consultation: A fusion of horizons. British Journal of General Practice, 1, 58-60.

Clark, S., \& Miles, M. (1999). Conflicting responses: The experiences of fathers of infants diagnosed with severe congenital heart disease. Journal of the Society of Pediatric Nurses, 4(1), 7-14.

Gadamer, H. G. (1975). Truth and method. (G. Barden \& J. T. Cumming, Trans.). New York: Seabury Press. (Original work published 1960)

Graungaard, A., \& Skov, L. (2006). Why do we need a diagnosis? Qualitative study of parents' experiences, coping and needs, when the newborn child is severely disabled. Child: Care, Health, \& Development, 33(3), 296-307.

Harden, J. (2005). Parenting a young person with mental health problems: Temporal disruption and reconstruction. Sociology of Health \& IIIness, 27(3), 351-371.

Heidegger,M. (1962). Being and time (J.Macquarie \& E. Robinson, Trans.). New York: Harper \& Row. (Original work published 1927)

Hill, V., Sahhar, M., Aitken, M., Savarirayan, R., \& Metcalfe, S. (2003). Experiences at the time of diagnosis of parents who have a child with a bon dysplasia resulting in short stature. American Journal of Medical Genetics, 122A, 100-107.

Holroyd, E. E. (2003). Chinese cultural influences on parental caregiving obligations toward children with disabilities. Qualitative Health Research, 13(1), 4-19.

Huang, Y. P., Kellett, U. M., \& St John, W. (2010). The experience of Taiwanese mothers after learning their child's diagnosis of cerebral palsy. Journal of Advanced Nursing, 66(6), 12131221.

Hwang, K. K. (1987). Face and favor: The Chinese power game. American Journal of Sociology, 92(4), 944-974. 
Kearney, P., \& Griffin, T. (2001). Between joy and sorrow: Being a parent of a child with developmental disability. Journal of Advanced Nursing, 34(5), 582-592.

Keller, D., \& Honig, A. S. (2004). Maternal and paternal stress in families with school-aged children with disabilities. American Journal of Orthopsychiatry, 74(3), 337-348.

Kellett, U. M. (1997). Heideggerian phenomenology: An approach to understanding family caring for an older relative. Nursing Inquiry, 4, 57-65.

Kerr, S., \&McIntosh, J. (1998). Disclosure of disability: Exploring the perspective of parents. Midwifery, 14, 225-232.

Koch, T. (1998). Story telling: Is it really research? Journal of Advanced Nursing, 28, 1182-1190.

Lai, A. (2006). Eye on religion: Cultural signs and caring for Chinese patients. Southern Medical Journal, 99(6), 688-689.

Lam, C. S., Tsang, H., Chan, F., \& Corrigan, P. W. (2006). Chinese and American perspectives on stigma. Rehabilitation Education, 20(4), 269-279.

Lee, E. (1999). Asian American families: An overview. In M. McGoldrick, J. Giordano, \& J. K. Pearce (Eds.), Ethnicity and family therapy (2nd ed., pp. 227-248). New York: The Guilford Press.

Lewis, C., Skirton, H., \& Jones, R. (2010). Living without a diagnosis: The parental experience. Genetic Testing and Molecular Biomarkers, 14, 807-816.

Lincoln, Y. S.,\& Guba, E. G. (1985). Naturalistic inquiry. Newbury Park, CA: Sage Publications.

Liu, G. Z. (2001). Chinese culture and disability: information for U.S. service providers. Retrieved September 15, 2010, from http://cirrie.buffalo.edu/monographs/china.php

Makela, N., Birch, P., Friedman, J., \& Marra, C. (2009). Parental perceived value of a diagnosis for intellectual disability (ID): A qualitative comparison of families with and without a diagnosis for their child's ID. American Journal of Medical Genetics, 149A, 2393-2402.

McGrath, P., Paton, M., \& Huff, N. (2004). Beginning treatment for paediatric acute myeloid leukaemia: Diagnosis and the early hospital experience. Scandinavian Journal of Caring Sciences, 18, 358-367.

McNeill, T. (2004). Fathers' experience of parenting a child with juvenile rheumatoid arthritis. Qualitative Health Research, 14(4), 526-545.

Meleis, A. L., Sawyer, L. M., Im, E. O., Hilfinger Messias, D. K., \& Schumacher, K. (2000). Experiencing transitions: An emerging middle-range theory. Advances in Nursing Science, 23(1), $12-28$.

Mri, G., \& Tovey, P. (2003). Asian carers' experiences of medical and social care: The case of cerebral palsy. British Journal of Social Work, 33, 465-479.

Palmer, R. E. (1969). Hermeneutics: Interpretation theory in Schleiermacher, Dilthey, Heidegger, and Gadamer. Evanston, IL: Northwestern University Press.

Park, M., \& Chesla, C. (2007). Revisiting Confucianism as a conceptual framework for Asia family study. Journal of Family Nursing, 13(3), 293-311.

Pelchat, D., Levert, M. J., \& Bourgeois-Gue' rin, V. (2009). How do mothers and fathers who have a child with disability describe their adaptation/transformation process. Journal of Child Health Care, 13(3), 239-259.

Ponterotto, J. G. (2006). Brief note on the origins, evolution, and meaning of the qualitative research concept "thick description." The Qualitative Report, 11, 538-549.

Premberg, A., Hellstrom, A. L., \& Berg, M. (2008). Experiences of the first year as father. Scandinavian Journal of Caring Sciences, 22, 56-63.

Rodgers, B. L., \& Cowles, K. V. (1993). The qualitative research audit trail: A complex collection of documentation. Research in Nursing \& Health, 16, 219-226.

Schuengel, C., Rentinck, I. C. M., Stolk, J., Voorman, J. M., Loots, G. M. P., Ketelaar, M., et al. (2009). Parents' reactions to the diagnosis of cerebral palsy: Associations between resolution, age and severity of disability. Child: Care, Health, and Development, 35(5), 673-680.

Schumacher, K. L., \& Meleis, A. L. (1994). Transition: A central concept in nursing. Journal of Nursing Scholarship, 26(2), 119-127.

Wojnar, D. M., \& Swanson, K. M. (2007). Phenomenology: An exploration. Journal of Holistic Nursing, 25(3), 172-180.

Yeo, S. S., Meiser, B., Barlow-Stewart, K., Goldstein, D., Tucker, K., \& Eisenbruch, M. (2005). Understanding community beliefs of Chinese-Australians about cancer: Initial insights using an ethnographic approach. Psycho-Oncology, 14, 174-186.

Yip, K. S. (2004). The empowerment model: A critical reflection of empowerment in Chinese culture. Social Work, 49, 479-487. 